\title{
The Predictive Value of Porcine Seminal Parameters on Fertility Outcome under Commercial Conditions
}

\author{
J Gadea, E Sellés and MA Marco \\ Department of Physiology, Faculty of Veterinary Medicine, University of Murcia, Spain
}

\section{Contents}

The aim of this study was to address the question of whether differences in farrowing rate and litter size after the use of different ejaculates could be predicted using the standard semen parameters under commercial conditions. In this study, a total of 1818 sows were used to evaluate the fertility predictive value of different sperm parameters. Logistic regression analysis (univariate and multivariate) was used to correlate the dichotomous farrowing rate data to the sperm parameters. Linear regression was also used to determine the relationship between litter size and semen parameters (Pearson correlation and multiple regression). Receiver-operating curves (ROC) were used to determine the overall performance characteristics of each semen variable in the logistic regression model. Semen analysis, under commercial conditions, allows to identify ejaculates with very low fertility potential but the pre-selection of the samples, the high number of sperm per doses and the high quality of the semen used in artificial insemination (AI) programmes reduces the variability. Therefore, it is unlikely to detect fertility differences associated with seminal parameters.

\section{Introduction}

The prediction of sperm fertilizing ability has a great economic importance to breeding herds when artificial insemination is used, as it leads to the selection of those boars with a good reproductive performance. However, at the moment, only a few trials that correlate sperm factors and fertility have been reported (reviewed by Gadea 2003). In most of them, a reduced or poor significant relationship has been observed (reviewed by Woelders 1991; Flowers 2002), in part, because of the many factors involved in fertility in sows (Foote 2003).

Classical methods of semen evaluation generally measure sperm concentration, progressive motility, the percentage of viable cells and acrosome morphology. These assays are poor in predicting fertility outcome, because only those samples with markedly poor quality can be identified (Gadea et al. 1998). To solve this problem, new procedures of seminal analysis in vitro (Braundmeier and Miller 2001), or multiple analysis of the same sample have been evaluated (Woelders 1991) and new assays to study the oocyte-sperm interaction as the in vitro fertilisation (IVF) system have been developed (Gadea et al. 1998; Xu et al. 1998; Selles et al. 2003). However, these analyses are expensive and timeconsuming, and cannot be applied under field commercial conditions.

Under field commercial conditions, the use of simple, non-expensive and accurate assays is required. Such goal has been pursued over decades but no definitive solution has ever been reported.
The aim of this study was to address the question of whether farrowing rate and litter size after the use of different ejaculates could be predicted with classical semen-quality parameters under commercial conditions. Different analytical strategies have been developed to determine the possible relationship between sperm parameters and farrowing rates and litter size. The first is the exploration of diffences in seminal parameters between ejaculates classified in two categories according to farrowing rate, with a target value for fertility under commercial conditions ( $85 \%$ farrowing rate). Secondly, logistic and linear regression analyses were applied to detect any significant relationship between semen quality and farrowing rate and litter size.

\section{Materials and Methods}

Spermatozoa from 273 ejaculates from 57 boars (four Dalland and 53 Pietrain) were evaluated for semenquality parameters in vitro. Sperm-rich fractions were collected by an experienced operator using the glovedhand technique. At the time of collection, the ejaculate was filtered through a gauze to remove gel and immediately placed at $37^{\circ} \mathrm{C}$ in a temperaturecontrolled water bath. Each ejaculate was later diluted to a final concentration of $3 \times 10^{7}$ spermatozoa $/ \mathrm{ml}$ in a commercial extender for refrigerated semen (MR-A@), Kubus, Madrid, Spain) and stored at $17^{\circ} \mathrm{C}$ until assay.

\section{Routine laboratory evaluation of semen}

The volume of the sperm-rich fraction of the ejaculate was determined in a graduated cylinder and sperm concentration measured with a haemacytometer. To evaluate the motility and the forward progressive motility (FPM), two subsamples were placed on warm glass slides $\left(39^{\circ} \mathrm{C}\right)$ and examined under a light microscope $(\times 400$ magnification). The percentage of motile sperm cells was estimated subjectively to the nearest $5 \%$ and the FPM using an arbitrary scale of $0-5$.

Wet mounts of semen fixed in buffered 2\% glutaraldehyde solution were examined under a phase-contrast microscope $(\times 1000$ magnification $)$ to analyse the morphology and acrosomes (Pursel et al. 1972). The proportion of spermatozoa with a normal apical ridge (NAR) was determined on two slides per sample and a total of 200 spermatozoa per sample. Two hundred spermatozoa were categorized according to sperm morphology into those with normal morphology, cells with attached proximal cytoplasmic droplets (PCD), distal 
cytoplasmic droplets (DCD), folded tail, coiled tail and others (abnormal heads) etc.

Eosin-nigrosin (EN) viability staining of sperm was applied. A semen sample was diluted $1: 1$ with staining solution $(5 \%$ yellow eosin, $10 \%$ nigrosin in a citrate solution $\mathrm{pH}$ 7.4) and smeared. Air-fixed stained spermatozoa were observed and 200 spermatozoa were evaluated per slide (Bamba 1988) and the percentage of non-stained spermatozoa was registered.

Sperm membrane integrity was assessed by incubation with carboxyfluorescein diacetate (DCF) (Harrison and Vickers 1990) on at least 200 cells/sample using a microscope equipped with epi-fluorescence.

\section{Fertility trial}

The fertility study was conducted on a commercial farm, using multiparous (two to six pregnancies) crossbred sows (Landrace $\times$ Large White). Oestrus was checked daily in the presence of a mature teaser boar. Occurrence of oestrus was defined by the standing reflex in front of a boar (back pressure test) and reddening and swelling of the vulva. The sows were inseminated with diluted semen, containing $3 \times 10^{9}$ sperm in $100 \mathrm{ml}$ MR-A extender, using disposable artificial insemination (AI) catheters. Insemination of all sows showing oestrus signs took place on immediately after the diagnosis of oestrus and was repeated $24 \mathrm{~h}$ later under the same conditions with semen preserved at $17^{\circ} \mathrm{C}$.

Fertility was measured for every ejaculate as the percentage of sows farrowing to AI. For each sow that farrowed, the number of dead and live piglets was counted and the sum was defined as the total number of piglets born (TPB).

\section{Experimental design}

In this study, a total of 1818 sows were used to evaluate the capacity for fertility prediction of the different sperm assays. Ejaculate was consider to be the experimental unit. First, the ejaculates were grouped into two categories according to fertility rate (low, farrowing rate $\leq 85 \%$; high, farrowing rate $>85 \%$ ). Seminal parameters were evaluated and differences between fertility groups were investigated. Secondly, logistic and linear regression analyses were applied to detect any significant relationship between semen quality and farrowing rate and litter size.

\section{Statistical analysis}

The results are expressed as mean \pm SEM and were analysed by one-way ANOVA, using the multivariate general linear models of Systat. When ANOva revealed a significant effect, values were compared by Tukey's test.

Logistic regression (univariate and multivariate) was used to relate the dichotomous fertility rate data to the sperm parameters. Linear regression was used to further investigate relationships between litter size and measured semen parameters (Pearson's correlation and multiple regression). Receiver-operating curves (ROC) were used to determine the overall discriminant power of each semen variable in the logistic regression model. A ROC was also used to calculate the elective breaking point (cut-off value) for each semen variable in relation to the fertility trial. The breaking point is in relation with the point that maximized specificity and sensitivity.

\section{Results \\ Relationship between sperm characteristics and farrowing rate}

Semen parameters values and the fertility outcome were not significantly different in the two genetic lines of the boars used in this study (data not shown). Therefore, all ejaculates from these boars can be considered as a homogeneous group. Statistical analysis of sperm parameters values and fertility outcome obtained in the field trial are shown in Table 1 for the two groups of ejaculates according to farrowing rate (more or less than

\begin{tabular}{|c|c|c|c|}
\hline & $\begin{array}{c}\text { Low fertility } \\
\text { (farrowing rate } \leq 85 \% \text { ) }\end{array}$ & $\begin{array}{c}\text { High fertility } \\
\text { (farrowing rate }>85 \% \text { ) }\end{array}$ & $\begin{array}{l}\text { Significance } \\
\text { (p-value) }\end{array}$ \\
\hline Number of ejaculates & 138 & 135 & \\
\hline Volume (ml) & $103.20 \pm 2.03$ & $107.13 \pm 2.09$ & 0.1792 \\
\hline Concentration $\left(10^{3}\right.$ sperm $\left./ \mathrm{mm}^{3}\right)$ & $77.91 \pm 2.48$ & $82.20 \pm 2.63$ & 0.2358 \\
\hline Total cells $\left(10^{9}\right)$ & $78.20 \pm 2.71$ & $86.10 \pm 2.82$ & 0.0443 \\
\hline Motility & $74.09 \pm 0.63$ & $75.31 \pm 0.63$ & 0.1760 \\
\hline FPM & $3.24 \pm 0.03$ & $3.20 \pm 0.03$ & 0.4669 \\
\hline EN (\%) & $84.72 \pm 0.70$ & $84.82 \pm 0.68$ & 0.9147 \\
\hline NAR $(\%)$ & $93.46 \pm 0.46$ & $93.56 \pm 0.43$ & 0.8755 \\
\hline $\mathrm{DCF}(\%)$ & $84.23 \pm 0.71$ & $84.30 \pm 0.87$ & 0.9510 \\
\hline Morphoanomalies (\%) & $11.94 \pm 0.78$ & $12.54 \pm 0.97$ & 0.6307 \\
\hline $\operatorname{PCD}(\%)$ & $2.73 \pm 0.27$ & $3.41 \pm 0.37$ & 0.1376 \\
\hline $\operatorname{DCD}(\%)$ & $4.22 \pm 0.38$ & $4.00 \pm 0.33$ & 0.6583 \\
\hline Folded tail $(\%)$ & $4.05 \pm 0.49$ & $3.73 \pm 0.69$ & 0.7002 \\
\hline Swollen tail $(\%)$ & $0.30 \pm 0.07$ & $0.75 \pm 0.30$ & 0.1325 \\
\hline Other anomalies $(\%)$ & $0.63 \pm 0.12$ & $0.64 \pm 0.12$ & 0.9691 \\
\hline Number of sows & 915 & 903 & \\
\hline Farrowing rate $(\%)$ & $645 / 915(70.49 \%)$ & $868 / 903(96.12 \%)$ & $<0.0001$ \\
\hline Live piglets born & $9.64 \pm 0.11$ & $9.84 \pm 0.10$ & 0.1689 \\
\hline Total piglets born & $10.25 \pm 0.12$ & $10.45 \pm 0.11$ & 0.2191 \\
\hline
\end{tabular}
for 273 ejaculates from 57 boars allocated into two groups according to farrowing rate $($ mean \pm SEM $)$

FPM, forward progressive motility (0-5); EN, eosin-nigrosin stain; NAR, normal apical ridge; DCF, sperm membrane integrity assessed with carboxyfluorescein diacetate; PCD, proximal cytoplasmic droplets; DCD, distal cytoplasmic droplets. 
Table 2. Univariate logistic and linear regression of seminal measurements with farrowing rate

\begin{tabular}{lccccc}
\hline & \multicolumn{2}{c}{ Logistic regression } & & \multicolumn{2}{c}{ Linear regression } \\
\cline { 2 - 3 } \cline { 5 - 6 } Parameter & & & & $\begin{array}{c}\text { Pearson } \\
\text { correlation }\end{array}$ & Significance \\
& Odd ratio & $\begin{array}{c}\text { Significance } \\
\text { (p-value) }\end{array}$ & & $\begin{array}{c}\text { coefficient }(\mathrm{r}) \\
\text { (p-value) }\end{array}$ \\
\hline Volume & 1.0017 & 0.5076 & & 0.0157 & 0.5073 \\
Concentration & 1.0016 & 0.4803 & & 0.0166 & 0.4828 \\
Total cells & 1.0029 & 0.1499 & & 0.0338 & 0.1530 \\
Motility & 1.0195 & 0.0237 & & 0.0539 & 0.0214 \\
FPM & 0.8764 & 0.4337 & & -0.0184 & 0.4322 \\
EN & 1.0013 & 0.8674 & & 0.0039 & 0.8673 \\
NAR & 1.0079 & 0.4891 & & 0.0164 & 0.4845 \\
DCF & 0.9993 & 0.9273 & & -0.0021 & 0.9274 \\
Morphoanomalies & 1.0009 & 0.8892 & & 0.0033 & 0.8896 \\
PCD & 1.0346 & 0.0621 & & 0.0421 & 0.0723 \\
DCD & 0.9986 & 0.9252 & & -0.0022 & 0.9252 \\
Folded tail & 0.9885 & 0.2300 & -0.0291 & 0.2138 \\
Swollen tail & 1.0768 & 0.0849 & 0.0324 & 0.1666 \\
Other anomalies & 0.9671 & 0.4862 & -0.0166 & 0.4784 \\
\hline
\end{tabular}

FPM, forward progressive motility (0-5); EN, eosin-nigrosin stain; NAR, normal apical ridge; DCF, sperm membrane integrity assessed with carboxyfluorescein diacetate; PCD, proximal cytoplasmic droplets; DCD, distal cytoplasmic droplets.

Table 3. Multiple logistic regression of semen measurements with farrowing rate (forward stepwise model)

\begin{tabular}{lrccrr}
\hline Parameter & Estimate & SE & Odds ratio & t-ratio & p-value \\
\hline Constant & -0.0873 & 0.6511 & - & -0.1341 & 0.8934 \\
Motility & 0.0209 & 0.0086 & 1.0211 & 2.4172 & 0.0156 \\
PCD & 0.0385 & 0.0194 & 1.0392 & 1.9843 & 0.0472 \\
Swollen tail & 0.0679 & 0.0516 & 1.0702 & 1.3147 & 0.1886 \\
\hline
\end{tabular}

$\mathrm{p}=0.0069 ;$ McFadden's $\rho^{2}=0.0075$.

PCD, proximal cytoplasmic droplets.

$85 \%$ ). Ejaculates were distributed equally in both groups $(\mathrm{n}=138$ vs 135$)$. The mean values of farrowing rate obtained in the two groups of sows $(n=915$ and 903) were significantly different $(70.49 \%$ vs $96.12 \%$, $\mathrm{p}<0.0001)$. No differences in semen parameters were found between both groups. Only total cell count in the ejaculate was significantly higher in the high fertility group $(\mathrm{p}=0.0443)$.

Linear and logistic regression analyses were used to correlate the farrowing rate data to the semen parameters. In both analyses, a poor correlation with fertility outcome was obtained. Only motility (logistic and linear regression) was significantly correlated to farrowing rate (Table 2). When all conventional semen parameters were analysed through a stepwise, multiple logistic or linear regression analysis, only three parameters (motility, PCD and swollen tail) are necessary to reach statistical significant model (Tables 3 and 4). However, in both models a low percentage of total variance of farrowing rate could be predicted. It was surprising to find that there was a positive correlation between PCD and swollen tail with fertility (Table 3 ).

The use of ROC led us to calculate the cut-off values for the semen parameters (Table 5) in these experimental conditions. Cut-off values obtained in this study were highly restricted, for example EN (86), NAR (94),
Table 4. Multiple linear regression of semen measurements with farrowing rate (forward stepwise model)

\begin{tabular}{lcccc}
\hline Parameter & Coefficient & SE & t-ratio & p-value (two-tail) \\
\hline Constant & 0.5867 & 0.0931 & 6.3032 & 0.0000 \\
Motility & 0.0031 & 0.0012 & 2.5035 & 0.0124 \\
PCD & 0.0046 & 0.0023 & 1.9622 & 0.0499 \\
Swollen tail & 0.0056 & 0.0034 & 1.6385 & 0.1015 \\
\hline
\end{tabular}

$\mathrm{p}=0.0090$; multiple $R^{2}=0.0063$.

PCD, proximal cytoplasmic droplets.

Table 5. Cut-off values (breaking points) of semen parameters significantly related to farrowing rate calculated from receiver-operating curves (ROC)

\begin{tabular}{lcccc}
\hline Variable & Cut-off value & Sensitivity & Specificity & Area ROC \\
\hline Volume & 100 & 52.9 & 49.2 & $0.508 \pm 0.018$ \\
Concentration & 77 & 51.5 & 51.8 & $0.509 \pm 0.018$ \\
Total cells & 84 & 50.7 & 50.5 & $0.518 \pm 0.018$ \\
Motility & 70 & 71.9 & 33.8 & $0.531 \pm 0.018$ \\
FPM & 3.5 & 87.9 & 13.9 & $0.510 \pm 0.018$ \\
EN & 86 & 53.0 & 45.4 & $0.499 \pm 0.018$ \\
NAR & 94 & 51.9 & 51.0 & $0.509 \pm 0.018$ \\
DCF & 85 & 51.8 & 49.7 & $0.504 \pm 0.018$ \\
Morphoanomalies & 10 & 53.8 & 48.6 & $0.501 \pm 0.018$ \\
PCD & 1 & 55.4 & 51.0 & $0.534 \pm 0.018$ \\
DCD & 3 & 54.3 & 44.0 & $0.501 \pm 0.018$ \\
Folded tail & 2 & 67.0 & 41.7 & $0.541 \pm 0.018$ \\
Swollen tail & 1 & 23.2 & 79.1 & $0.512 \pm 0.018$ \\
Other anomalies & 1 & 71.6 & 28.1 & $0.500 \pm 0.018$ \\
\hline
\end{tabular}

FPM, forward progressive motility (0-5); EN, eosin-nigrosin stain; NAR, normal apical ridge; DCF, sperm membrane integrity assessed with carboxyfluorescein diacetate; $\mathrm{PCD}$, proximal cytoplasmic droplets; $\mathrm{DCD}$, distal cytoplasmic droplets.

normal morphology (90) and DCF (85). However, they were less restricted for motility (70). The sensitivity and specificity of these parameters to identify better ejaculates in terms of fertility outcome are shown in Table 5. Lowering the threshold reduces the sensitivity of the measures (the likelihood of correctly identifying infertile-subfertile ejaculates) but increases their specificity (the likelihood of correctly identifying fertile ejaculates).

On the basis of the area under ROC (area ROC), each seminal parameter did not provide information that was helpful in discriminating fertility outcome, because it was not significantly $>0.500$. However, motility, PCD and folded tail are near to this value, although there were not significant differences between area ROC corresponding to the different seminal parameters.

\section{Relationship between sperm characteristics and litter size}

Concerning litter size, the total number of piglets born showed a significant positive correlation with motility $(\mathrm{r}=0.1223, \mathrm{p}=0.0435$; Table 6$)$, while folded tail showed a negative correlation $(\mathrm{p}=0.0681)$ but did not reach statistical significance. However, no correlation was found between the average live piglets born and semen parameters (Table 6). Only a weak positive correlation was found for motility and a negative correlation for folded tail, but neither reach statistical significance. 
Table 6. Linear regression of seminal measurements with average litter size ${ }^{\mathrm{a}}$

\begin{tabular}{|c|c|c|c|c|}
\hline & \multicolumn{2}{|c|}{ Live piglets born } & \multicolumn{2}{|c|}{ Total piglets born } \\
\hline & $\begin{array}{l}\text { Correlation } \\
\text { coefficient (r) }\end{array}$ & p-value & $\begin{array}{l}\text { Correlation } \\
\text { coefficient (r) }\end{array}$ & $\mathrm{p}$-value \\
\hline Volume & -0.0398 & 0.5149 & -0.0650 & 0.2872 \\
\hline Concentration & 0.0945 & 0.1213 & 0.1027 & 0.0921 \\
\hline Total cells & 0.0798 & 0.1909 & 0.0716 & 0.2406 \\
\hline Motility & 0.1143 & 0.0592 & 0.1223 & 0.0435 \\
\hline FPM & -0.0557 & 0.3594 & -0.0541 & 0.3728 \\
\hline EN & 0.0578 & 0.3413 & 0.0705 & 0.2454 \\
\hline NAR & -0.0044 & 0.9419 & -0.0062 & 0.9193 \\
\hline $\mathrm{DCF}$ & 0.0637 & 0.2959 & 0.0513 & 0.4001 \\
\hline Morphoanomalies & -0.0610 & 0.3150 & -0.0673 & 0.2679 \\
\hline PCD & 0.0531 & 0.3819 & 0.0522 & 0.3905 \\
\hline $\mathrm{DCD}$ & -0.0388 & 0.5232 & -0.0565 & 0.3528 \\
\hline Folded tail & -0.1179 & 0.0516 & -0.1106 & 0.0681 \\
\hline Swollen tail & 0.0574 & 0.3444 & 0.0502 & 0.4085 \\
\hline Other anomalies & 0.0065 & 0.9149 & -0.0075 & 0.9021 \\
\hline
\end{tabular}

${ }^{a}$ Litter sizes used included failed conceptions as zero values.

FPM, forward progressive motility $(0-5)$; EN, eosin-nigrosin stain; NAR, normal apical ridge; DCF, sperm membrane integrity assessed with carboxyfluorescein diacetate; PCD, proximal cytoplasmic droplets; DCD; distal cytoplasmic droplets.

Table 7. Multiple linear regression (stepwise forward) of seminal measurements with average number of piglets live ${ }^{a}$

\begin{tabular}{lrrrr}
\hline Variable & Coefficient & SE & t-ratio & p-value \\
\hline Constant & 7.9670 & 1.5577 & 5.1146 & 0.0000 \\
Motility & 0.0302 & 0.0174 & 1.7378 & 0.0834 \\
FPM & -0.6110 & 0.3517 & -1.7376 & 0.0834 \\
Folded tail & -0.0317 & 0.0184 & -1.7275 & 0.0852 \\
\hline
\end{tabular}

Multiple $R^{2}: 0.0318, \mathrm{p}=0.0335$

${ }^{a}$ Litter sizes used included failed conceptions as zero values.

Table 8. Multiple linear regression (stepwise forward) of seminal measurements with average total number of piglets ${ }^{\mathrm{a}}$

\begin{tabular}{lrrrr}
\hline Variable & Coefficient & SE & t-ratio & p-value \\
\hline Constant & 7.9637 & 1.6906 & 4.7107 & 0.0000 \\
Motility & 0.0367 & 0.0186 & 1.9707 & 0.0498 \\
FPM & -0.6109 & 0.3755 & -1.6268 & 0.1050 \\
PCD & 0.0645 & 0.0392 & 1.6452 & 0.1011 \\
Swollen tail & 0.0921 & 0.0548 & 1.6814 & 0.0938 \\
Morphoanomalies & -0.0286 & 0.0159 & -1.8007 & 0.0729 \\
\hline
\end{tabular}

Multiple $R^{2}: 0.0417, \mathrm{p}=0.0435$.

${ }^{\mathrm{a}}$ Litter sizes used included failed conceptions as zero values.

Significant models were found with multiple linear regression (stepwise forward). For the live piglets born, the model was constructed with three variables including motility, FPM and folded tail, confirming the tendency previously detected (Table 7). For total number of piglets born, a significant model was constructed with five parameters (motility, FPM, PCD, swollen tail and total morpho-anomalies (Table 8). In both models, a low percentage of total variance of the litter size could be predicted.

\section{Discussion}

Fertilizing ability is commonly measured as the percentage of sows or gilts conceiving or farrowing after AI (Foote 2003). In general, both measures are indicative of the efficiency with which eggs are fertilized and capable of sustaining embryonic development (Watson 1996). The best way to assess boar fertility is to obtain viable pregnancies and normal offspring following in vivo insemination. However, field trials of semen fertility are imprecise (Clark et al. 1989; Foote 2003). Consequently, one of the main goals in spermatology should be to investigate new markers of sperm function that would allow the better prediction of fertility outcome (Hammerstedt 1996; Braundmeier and Miller 2001).

Several studies have previously assessed the predictive value of seminal parameters under different experimental conditions, including insemination with low number of sperm per dose (Tardif et al. 1999); no previous selection of ejaculates (Gadea et al. 1998), one or two AI doses, different time application, etc. However, this study was designed to provide sound scientific information that could be directly applied in the field, under commercial conditions ( 2 artificial insemination (AI), $3 \times 10^{9} \mathrm{sperm} /$ dose, pre-selection of ejaculates). So, we grouped the ejaculates according to the farrowing rate, and selected the threshold value of $85 \%$ because it is a common target value for a great numbers of commercial farms.

In this study a poor correlation was found between semen characteristics and fertility outcome. Of all parameters studied, sperm motility is the most significant one because it is significantly correlated with farrowing rate and total number of piglets born, and it appears as a significant component in all multivariate models. However, the correlation coefficient is relatively low. Reports of a correlation between motility and fertility are contradictory (Pursel et al. 1984; Galli and Bosisio 1988; Berger and Parker 1989; Holt et al. 1997; Gadea et al. 1998; Tardif et al. 1999; Selles et al. 2003). This could be due to differences in experimental conditions. In fact, sperm motility assessment is subject to great intra- and inter-observer variability. Although computer-assisted sperm analyses (CASA) would appear as the logical choice to solve this question (Holt et al. 1997), CASA is under other technical biases.

Sperm morphology is another parameter that appears to be correlated with fertility outcome (at least as a tendency). Sperm defects have been related to infertility (Bonet and Briz 1991). In a standard semen analysis, morphology provides information about the efficiency of spermatogenesis and it can facilitate selection of boars for AI programmes (Waberski et al. 1990). It also allows workers to establish the intensity of stress produced by a high frequency of semen collection better (Briz et al. 1995). An inverse correlation has been reported between the number of morphological abnormalities and fertility (Martinez et al. 1986; Galli and Bosisio 1988; Waberski et al. 1990; Zeuner 1992; Waberski et al. 1994a; Gadea et al. 1998). However, in this study under commercial conditions, only a weak correlation was found for some sperm defects (PCD, folded tail) but did not reach statistical significance. 
The intact nature of the spermatozoa plasma membrane is a prerequisite for suitable sperm metabolism and function. The methods used to assess sperm viability included EN staining and DCF. However, this information is not much related to fertility, perhaps because it informs about the viability of this sperm but not about its functionality, (capacitation process, acrosome reaction, sperm binding, etc.). Several authors have described how high numbers of altered acrosomes are related to fertilizing problems. However, the correlation rates observed between NAR and fertility were not high (Pursel et al. 1984; Galli and Bosisio 1988; Hammitt et al. 1989).

The use of multivariate analysis would help to discriminate fertility potential, because it combines information of different sperm properties. The combination of selected semen tests provides a higher fertility predictive value compared with single tests (Waberski et al. 1999). However, the results obtained in this study could only explain the low variance observed. Likewise, the use of logistic regression analysis (more adequate to dichotomous variables as farrowing rate) is equally efficient in correlating seminal parameters. As expected, there was a significant correlation between the different methods used confirming that 'biological evidence is related with facts, no with any peculiar statistical test' (A. Romar, personal communication).

The determination of cut-off values for seminal parameters has been described in the literature more as the result of an empirical than a scientific approach to the problem. A statistical discriminant analysis of the data could give some valuable information. However, because of the poor correlation between seminal parameters and fertility, the sensibility and specificity of this cut-off is very limited.

Some possible causes of the lack of correlation between fertility and seminal characteristic could be based on: (1) certain sperm characteristics could not be analysed by the standard spermiogram. In this sense, Saacke et al. (1994) define the uncompensable sperm traits as sperm characteristics or deficiencies associated with incompetent fertilizing sperm (those sperm that can initiate but not complete the fertilization process or sustain early embryogenesis). The compensable sperm traits precluded availability of sperm to fertilization (reviewed by Evenson 1999), and they could be measured by classical spermiogram. These uncompensable sperm traits would be in relation with alteration in DNA and nucleoproteins in sperm chromatin (Evenson et al. 1994).

(2) As the contradictory results would have been due to the experimental differences including low number of ejaculates or sows analysed, a high number of sperm per dose could compensate any infertility factor in the boar that could mask the relationship with sperm quality (Woelders 1991; Tardif et al. 1999; Johnson et al. 2000). Another factor could be the pre-selection of the ejaculates. As, under commercial conditions, the ejaculates were selected usually by motility, only those with motility over a reference value were used. This would tend to reduce the variability in semen parameters. In a previous study, in which no pre-selection was performed, higher correlations were found (Gadea et al. 1998), but the preselection carried out on commercial conditions could reduce this correlation, as demonstrated for in vitro fertility (Gadea and Matas 2000).

(3) A high variance associated with the sow was not related to sperm quality and was affected by many different factors (Clark et al. 1989). The higher is the sperm quality, the more homogeneous the sow effect is. One way of eliminating this variable would be to improve those factors that impinge on the sows such as the synchrony between inseminations and ovulation with the use of ultrasonography (Waberski et al. 1994b; Nissen et al. 1997).

In conclusion, sperm analysis conducted under commercial conditions lead to the detection of ejaculates with very poor quality (associated with poor fertility), but the pre-selection of the samples, the high number of sperm per dose and the high quality of the semen used in the AI programmes reduces the variability. So, there is low probability of detecting fertility differences associated with seminal parameters.

\section{Acknowledgements}

The authors thank Dr A. Romar for statistical review, Dr J. Alvarez for critical reading of the manuscript and $\mathrm{P}$. Tomás for recording the fertility data. This work was supported by project AGL2000-0485-C02-01 and AGL2003-03144.

\section{References}

Bamba K, 1988: Evaluation of acrosomal integrity of boar spermatozoa by bright field microscopy using a eosinnigrosin stain. Theriogenology 29, 1245-1251.

Berger T, Parker K, 1989: Modification of the zona free hamster ova bioassay of boar sperm fertility and correlation with in vivo fertility. Gamete Res 22, 385-397.

Bonet S, Briz MD, 1991: New data on aberrant spermatozoa in the ejaculate of Sus domesticus. Theriogenology 35, 725730.

Braundmeier AG, Miller DJ, 2001: Accurate molecular markers of male fertility: where do we go from here? IETS Newslett 19, 4-10.

Briz MD, Bonet S, Pinart B, Egozcue J, Camps R, 1995: Comparative study of boar sperm coming from the caput, corpus, and cauda regions of the epididymis. J Androl 16, 175-188.

Clark LK, Schinckel AP, Singleton WL, Einstein ME, Teclaw RF, 1989: Use of farrowing rate as a measure of fertility of boars. J Am Vet Med Assoc 194, 239-243.

Evenson DP, 1999: Loss of livestock breeding efficiency due to uncompensable sperm nuclear defects. Reprod Fertil Dev 11, 1-15.

Evenson DP, Thompson L, Jost L, 1994: Flow cytometric evaluation of boar semen by the sperm chromatin structure assay as related to cryopreservation and fertility. Theriogenology 41, 637-651.

Flowers WL, 2002: Increasing fertilization rate of boars: Influence of numer and quality of spermatozoa inseminated. J Anim Sci 80(E. Suppl. 1), 47-53.

Foote RH, 2003: Fertility estimation: a review of past experience and future prospects. Anim Reprod Sci 75, 119-139.

Gadea J, 2003. Sperm factors related to in vitro and in vivo porcine fertility. 5th International Conference on Boar Semen Preservation, Doorwerth, The Netherlands.

Gadea J, Matas C, 2000: Sperm factors related to in vitro penetration of porcine oocytes. Theriogenology 54, 13431357. 
Gadea J, Matás C, Lucas X, 1998: Prediction of porcine semen fertility by homologus in vitro penetration (hIVP) assay. Anim Reprod Sci 54, 95-108.

Galli A, Bosisio M, 1988: Quality of semen stored at +15 / $16^{\circ} \mathrm{C}$ is related to fertility of artificially inseminated swine. Theriogenology 30, 1185-1190.

Hammerstedt RH, 1996: Evaluation of sperm quality: identification of the subfertile male and courses of action. Anim Reprod Sci 42, 77-87.

Hammitt DG, Martin PA, Callanan T, 1989: Correlations between heterospermic fertility and assays of porcine seminal quality before and after cryopreservation. Theriogenology 32, 385-399.

Harrison RAP, Vickers SE, 1990: Use of fluorescent probes to assess membrane integrity in mammalian spermatozoa. J Reprod Fertil 88, 343-352.

Holt C, Holt WV, Moore HDM, Reed HCB, Curnock RM, 1997: Objectively measured boar sperm motility parameters correlate with the outcomes of on farm inseminations: results of two fertility trials. J Androl 18, 312-323.

Johnson LA, Weitze KF, Fiser P, Maxwell WMC, 2000: Storage of boar semen. Anim Reprod Sci 62, 143172.

Martinez E, Ruiz S, Sebastian J, Sanchez R, Garcia C, Martin S, 1986: Factores que afectan a la inseminación artificial porcina. An Vet (Murcia) 2, 115-120.

Nissen K, Soede NM, Hyttel P, Schmidt M, D'Hoore L, 1997: The influence of time of insemination relative to time of ovulation on farrowing frequency and litter size in sows, as investigated by ultrasonography. Theriogenology 47, 15711582.

Pursel VG, Johnson LA, Rampacek GB, 1972: Acrosome morphology of boar spermatozoa incubated before cold shock. J Anim Sci 34, 278-283.

Pursel VG, Rexroad CE, Wall RJ, 1984: Relationship of competitive fertility to quality of boar semen. Proc 10th Int Congr Anim Reprod Artif Insem 2, 63-65.

Saacke RG, Nadir S, Nebel RL, 1994: Relationship of semen quality to sperm transport, fertilization and embryo quality in ruminants. Theriogenology 41, 45-50.

Selles E, Gadea J, Romar R, Matas C, Ruiz S, 2003. Analysis of in vitro fertilizing capacity to evaluate the freezing procedures of boar semen and to predict the subsequent fertility. Reprod Domest Anim 38, 66-72.

Tardif S, Laforest JP, Cormier N, Bailey JL, 1999: The importance of porcine sperm parameters on fertility in vivo. Theriogenology 52, 447-459.

Waberski D, Dirksen G, Weitze KF, Leiding C, Hang R, 1990: Field studies of the effect of sperm motility and morphology on the fertility of boars used for insemination (in German). Tierärztl Prax 18, 591-594.

Waberski D, Weitze KF, Gleumes T, Schwarz M, Willmen T, Petzoldt R, 1994a: Effect of time of insemination relative to ovulation on the fertility with liquid and frozen boar semen. Theriogenology 42, 831-840.

Waberski D, Weitze KF, Lietmann C, Lübber Zur Lage W, Bortolozzo FP; Willmen T, Petzoldt R, 1994b: The initial fertilizing capacity of long-term-stored liquid boar semen following pre- and post-ovulatory insemination. Theriogenology 41, 1367-1377.

Waberski D, Petrounkina A, Weitze KF, Topfer-Petersen E, 1999: In vitro assessment of semen for the prediction of fertility (in German). Tierarztl Prax Ausg G Grosstiere Nutztiere 27, 1-7.

Watson PF, 1996: Cooling of spermatooza and fertilizing capacity. Reprod Dom Anim 36, 135-140.

Woelders H, 1991: Overview of in vitro methods for evaluation of semen quality. In: Johnson LA, Rath D (eds), Boar Semen Preservation II. Paul Parey Scientific Publishers, Berlin and Hamburg, pp. 145-164.

Xu X, Pommier S, Arbov T, Hutchings B, Sotto W, Foxcroft GR, 1998: In vitro maturation and fertilization techniques for assessment of semen quality and boar fertility. J Anim Sci 76, 3079-3089.

Zeuner A, 1992: On the relations between sperm morphology and the fertility of boar semen. 12th Int Congr Anim Reprod, The Hague, The Netherlands vol. 3, pp. 16171619.

Submitted: 12.11 .2003

Author's address (for correspondence): Joaquín Gadea, Dept. Fisiología, Facultad de Veterinaria, Universidad de Murcia, 30.100 Murcia, Spain. E-mail: jgadea@um.es 